\title{
Las causas de la crisis desde la perspectiva de la Teoría de los Ciclos Reales
}

\author{
Jorge Turmo Arnal \\ Departamento de Análisis Económico: Teoría e Historia \\ Universidad Autónoma de Madrid
}

\section{INTRODUCCIÓN}

La crisis económica ha golpeado fuertemente a nuestro país. Hace un año, día por día, la Economía española crecía a un 3,5\% en tasa interanual. Hoy la tasa interanual en el tercer trimestre de 2008 es del 0,9\% y el Banco de España ha confirmado que, con los datos provisionales del cuarto trimestre, la economía española ha entrado en recesión. Las previsiones acerca del futuro se hacen más y más sombrías conforme pasa el tiempo y la estimación de la duración de nuestra crisis aumenta también. Las previsiones de Enero de 2009 del Ministerio de Economía son de una contracción del PIB del 1,6\% para este año. La Comisión Europea indica que la contracción será del $2 \%$. Por lo tanto el trabajo se acumula en la mesa de los economistas. Una de las tareas pendientes es la revisión de nuestro conocimiento adquirido sobre las crisis. Lo que ya vamos sabiendo sobre la crisis y los datos de que disponemos, si bien provisionales, deben llevarnos a analizar hasta qué punto nuestros modelos explicativos siguen vigentes o deben ser revisados. Una buena teoría es condición necesaria, aunque no suficiente, para manejar los retos que se plantean en todos los casos, pero más aún cuando son de la envergadura de los presentes.

El marco estándar para explicar las fluctuaciones cíclicas de la actividad económica es la Teoría de los Ciclos Reales. Aunque criticada desde algunas instancias, casi todos los expertos en fluctuaciones y crisis trabajan dentro de su marco metodológico por lo que debe ser considerada como la base principal para explicar lo 
que está ocurriendo ${ }^{1}$. La actual crisis pone a prueba su capacidad explicativa como pondremos de manifiesto a lo largo del trabajo y por tanto apunta a la necesidad de cambios y reformas, algunos de ellos muy profundos.

En la siguiente sección explicamos muy brevemente el surgimiento de la Teoría de los Ciclos Reales (RBC en abreviatura que utilizaremos) y su planteamiento general y ponemos de manifiesto cómo la teoría hace hincapié en los factores reales como causantes de las fluctuaciones, y más concretamente de de los shocks tecnológicos. En la tercera parte revisamos brevemente las principales fuentes de las fluctuaciones económicas tal y cómo las contempla la Teoría de los Ciclos Reales. A partir de la identificación de las causas del ciclo que la teoría proporciona analizamos los datos disponibles para España para establecer la importancia que esas variables causantes han tenido en el origen de la crisis cíclica en nuestro país. En la cuarta parte presentamos nuestras conclusiones a partir de los datos y las teorías de la parte tres, así como las sugerencias de cara a futuras líneas de investigación.

\section{La Teoría de los Ciclos Reales}

En su artículo fundamental de 1982 Kydland y Prescott ${ }^{2}$ sentaron las bases de un nuevo acercamiento al análisis de las fluctuaciones cíclicas de la economía. Partieron de la base de que el ciclo económico y el crecimiento eran dos partes de la misma dinámica y que por lo tanto el estudio de las fluctuaciones cíclicas tenía que partir de la tendencia de crecimiento que las principales economías occidentales mostraban. Al hacerlo Kydland y Prescott seguían la definición de Lucas (1977) según la cual el ciclo es la desviación de la producción total con respecto a su tendencia. De esta manera rompían con una larga tradición en la que el ciclo era analizado como un fenómeno en sí mismo, con sus fases, siempre las mismas y relativamente regulares ${ }^{3}$.

En su enfoque la tendencia de las series representa su evolución a largo plazo mientras que las desviaciones de la misma son la parte cíclica, que es lo que debe ser ex-

${ }^{1}$ Las explicaciones alternativas, como la keynesiana o la austriaca concitan mucho menos apoyo entre la profesión.

2 Kydland, F y Prescott, E. (1982), « Time to build and Aggregate Fluctuations»,

${ }^{3}$ Esta concepción fue la dominante a lo largo del siglo XX y su mayor exponente son los trabajos de Burns y Mitchell (1946). 
plicado. Por tanto se asume que la economía tiene un movimiento tendencial ${ }^{4}$ que es interrumpido o disturbado por la aparición de perturbaciones de varios tipos que hacen que de forma temporal la economía evolucione fuera de su tendencia para volver después a ella. El análisis se realiza con un modelo dinámico de equilibrio general en el que agentes racionales toman decisiones acerca de las decisiones de consumo y ocio que maximizan su utilidad y responden ante las variaciones de su entorno reajustando sus elecciones.

La especificación de las funciones de preferencia de los agentes y de la tecnología de la producción permitió a Kydland y Prescott utilizar los datos de la economía de Estados Unidos para aproximar los valores de los parámetros y comparar los resultados con los reales de la muestra de Estados Unidos entre 1950 y 19795. Las conclusiones que obtuvieron son ampliamente conocidas en términos cuantitativos, pero lo más notable era que la evolución de las principales variables del modelo era muy parecida a los datos empíricos de que disponían, mostrando que la especificación que habían realizado era muy adecuada. El modelo les permitía analizar con éxito los comovimientos de las series así como la persistencia de las mismas y mostraba valores de la volatilidad relativa y de correlación muy cercanos a los observados. Como consecuencia de todo esto la Teoría de los Ciclos Reales ( RBC en la terminología al uso) se estableció como un estándar en el análisis de las fluctuaciones cíclicas y muchos trabajos posteriores, partieron de ella para refinar y mejorar la comprensión del fenómeno.

La gran mayoría de los autores que estudian el ciclo económico lo hacen en el marco de la metodología RBC aunque discrepen en determinados aspectos. Por esa razón nuestro análisis de la crisis actual parte del mismo marco para estudiar si dentro de él es posible explicar lo que está ocurriendo teniendo en cuenta los datos de los que disponemos. Antes de abordar el análisis explícito de la cuestión vamos a hacer referencia a dos cuestiones. Por un lado la RBC distingue, como cualquier teoría del ciclo económico entre el impulso y el mecanismo de transmisión del ciclo. El mecanismo de transmisión son las decisiones de los agentes en cuanto a sus elecciones de consumo, trabajo y ocio a la vista de la información de que disponen. Se da por supuesto que las elecciones de los agentes son óptimas en todos los casos y que tan sólo una modificación en las condiciones en las que se desarrolla la actividad económica les obligan a revisarlas y

${ }^{4}$ En cierto modo corresponde al concepto de «Estado estacionario» en los trabajos de Solow (1956 y 1957).

5 Hodrick, R.J. y Prescott, E. (1980), « Post-War U.S. Business Cycles: An Empirical Investigation» Carnegie Mellon. 
que disponen de la información necesaria para tomar esas decisiones. Los cambios en las decisiones de trabajo, ocio, consumo y ahorro a nivel microeconómico generan una modificación en los agregados macroeconómicos que son los que se observan.

En el modelo RBC el impulso de las fluctuaciones son las perturbaciones del tipo que sea. Si no hay perturbaciones, o si éstas son de pequeña escala, la economía se mantiene en su tendencia y los agentes no modifican sus elecciones. Por tanto el ciclo sólo se produce cuando hay perturbaciones de la suficiente importancia, no es un fenómeno constante y recurrente. En realidad en la literatura se habla de que una serie «presenta mucho ciclo» refiriéndose a que el grado de desviación con respecto a la tendencia es grande. Por tanto la definición y medida de la tendencia resulta de gran importancia ${ }^{6}$. Nosotros estamos interesados aquí en las fuentes de las perturbaciones, el impulso del ciclo, que es lo que vamos a analizar para el caso de España en el presente.

Además la teoría de los ciclos reales recibe su nombre en gran medida del rechazo a considerar los fenómenos monetarios como una de las causas del ciclo y su hincapié en buscar siempre causas en la economía real. Como comentan Kydland y Prescott (1994):

There is no evidence that either the monetary base or M1 leads the cycle, although some economists still believe this monetary myth. Both the monetary base and M1 series are generally procyclical and, if anything, the monetary base lags the cycle slightly (1994:12).

La Teoría de los Ciclos Reales hace mucho hincapié en que tan sólo los fenómenos reales pueden generar ciclos, aunque en algunas ocasiones los elementos monetarios jueguen un papel reducido. No obstante en algunos años se han interesado en los posibles factores monetarios en los ciclos económicos.

Mi interés se centra en analizar los distintos tipos de perturbaciones que la Teoría de los Ciclos Reales considera como posibles fuentes del ciclo económico y estudiar con los datos disponibles para España la importancia relativa de cada una de esas fuentes en nuestro caso. ${ }^{7}$ Cuando el ciclo económico es de pequeña amplitud y se desvía poco de la tendencia del PIB el origen del mismo tiene una reducida importancia teórica

${ }^{6}$ No entraremos aquí en esta cuestión. Hay distintas maneras de calcular la tendencia y por tanto el ciclo.

${ }^{7}$ Las críticas Keynesianas a este enfoque como puede verse en Mankiw (1989) no inciden tanto en las posibles causas del ciclos sino en el mecanismo de transmisión en la economía y por 
pero cuando como en el caso actual hemos pasado de una tasa de crecimiento del PIB superior al 3\% a una situación de práctica recesión en tan sólo un año ${ }^{8}$ el origen es lo más importante, no tanto cómo reaccionan los agentes económicos y cómo se reajusta la economía. En términos de Política Económica necesitamos acertar con las recetas para salir de la crisis, pero en términos de Teoría Económica lo que precisamos es una explicación de porqué se ha producido9 .

\section{ANÁLISIS DE LAS CAUSAS DE LA CRISIS SEGÚN LA TeOría de los Ciclos ReAleS}

\subsection{Los shocks tecnológicos}

En los orígenes de la RBC está la consideración de que son los shocks tecnológicos, que no pueden anticiparse, los que provocan las fluctuaciones de la economía. En su trabajo de $1986^{10}$ Prescott afirma:

The finding that when uncertainty in the rate of technological change is incorporated into the growth model it displays the business cycle phenomena was both dramatic and unanticipated... (1986:12).

El cambio tecnológico lo mide Prescott siguiendo a Solow (1957) como la diferencia entre la variación de la producción agregada y la variación de los factores de producción multiplicada por la ponderación de esos factores en la función de producción. Es lo que se conoce como la Productividad Total de los Factores, que se utiliza de forma profusa para tener en cuenta el crecimiento de la producción que no depende directamente de la cuantía de los factores. Las estimaciones de ese factor para la eco-

tanto en la conveniencia o no de utilizar la política económica para corregir los problemas. Por esta razón no las consideramos.

${ }^{8}$ Cuando se escriben estas líneas disponemos del dato de decrecimiento del PIB en el 3er trimestre de 2008 , pero no del $4^{\circ}$, por lo que no puede hablarse en puridad de recesión.

9 Otra cuestión muy interesante es hasta qué punto la Política Económica ayuda en esta situación. De la Teoría de los Ciclos Reales se deduce que la intervención es contraproducente puesto que el ciclo es un fenómeno de equilibrio o ajuste a un nuevo equilibrio, no de desequilibrio.

${ }^{10}$ Prescott, E. Theory Ahead of Business Cycle Measurement. 
nomía de Estados Unidos entre 1955 y 1984 le adjudicaban un peso del 1,2\% en el crecimiento de USA. Por tanto de los datos se deducía que la Productividad Total de los Factores tenía gran importancia en el crecimiento de los factores y ese cambio técnico es al que apunta Prescott y la teoría de los ciclos reales como el principal causante de las fluctuaciones de la economía. Partiendo de los datos de Solow y refinándolos Prescott llega a la conclusión de que la desviación estándar de los shocks de cambio técnico está en 0.763 (Prescott 1986:18).

La magnitud de los shocks tecnológicos necesarios para obtener valores como los que Prescott mostró llevó a una doble matización de su planteamiento. Por un lado algunos autores pusieron de manifiesto que dado que la PTF es el residuo de Solow, en realidad es una medida de nuestra ignorancia y adjudicar su cambio a factores exclusivamente exógenos, como hace Prescott, no era correcto ${ }^{11}$. Era muy plausible que hubiese factores endógenos que explicasen parte de los cambios en la Productividad Total de los Factores, de forma que el efecto de los shocks externos podía ser más pequeño. Además y sobre todo, era bastante cuestionable el efecto de los shocks tecnológicos en las recesiones. El comportamiento de la producción por encima de su tendencia puede razonablemente deberse a un shock tecnológico positivo, pero es más difícil justificar que los datos de producción por debajo de la misma se deban a un shock tecnológico negativo, puesto que implicaría una regresión tecnológica.

Estas razones llevaron a varios teóricos a estudiar si el comportamiento de variables del sistema, y por tanto endógenas, podía amplificar el efecto de los shocks tecnológicos. Es decir, se trataba de analizar si pequeños shocks tecnológicos, pequeñas variaciones en la Productividad Total de los Factores, combinados con modificaciones en otros factores podían dar cuenta de los datos observados. De esa manera el cambio en la Productividad Total de los Factores sería el efecto conjunto de un shock tecnológico y de elementos como el grado de utilización del capital y el trabajo.

Galí ${ }^{12}$ plantea en su trabajo de 1999 una crítica fundamental a la importancia de los shocks tecnológicos para explicar las fluctuaciones cíclicas. Parte de un problema básico de los modelos de RBC: la predicción de que las horas trabajadas y la productividad han de tener una fuerte correlación. Esta predicción no se corresponde con los datos que muestran una correlación muy cercana a cero e incluso negativa en algunos casos. En lugar de intentar reconciliar los modelos de RBC con los datos en este punto, Galí plantea un modelo diferente que, siguiendo la metodología general contiene elementos

11 Al respecto puede consultarse Hall (1988) o Evans (1992).

12 Galí, J. (1999). 
claramente keynesianos. En su modelo se da competencia monopolística, precios rígidos y un nivel de esfuerzo variable por parte de los trabajadores.

Pero lo más importante es que Galí analiza los efectos sobre una economía definida sobre esas bases de los shocks tecnológicos y los no tecnológicos, entre los que Galí elije los monetarios. En su modelo la cantidad de dinero existente en la economía crece a un ritmo proporcional al del crecimiento del nivel tecnológico de la economía, matizado por un parámetro $\gamma$ que mide la respuesta de la autoridad monetaria al cambio tecnológico. Ante cualquier cambio tecnológico la cantidad de dinero también cambia. Esta modelización permite introducir un shock monetario, además del shock tecnológico real. Los resultados de Galí al respecto son sorprendentes: En el caso de un shock de origen tecnológico la productividad y las horas trabajadas evolucionan en sentidos opuestos y el comovimiento es negativo, mientras que cuando se da un shock monetario ambas variables cambian en la misma dirección y el comovimiento es positivo. En el agregado, y teniendo en cuenta ambos shocks lo que de su modelo se deduce es que la relación entre la productividad y las horas trabajadas está en el entorno de cero, que es lo que los datos muestran.( Galí: 250). La principal conclusión de Galí es que:

...whether the technology shocks in actual economies are responsible for the pattern of GDP and labor-input fluctuations associated with business cycles remains and open question, and one which should provide a critical test of the relevance of a research program that aims to interpret the bulk of aggregate fluctuations as resulting from those shocks (Galí, 1999:265).

El análisis de Galí ha sido cuestionado por otros expertos en Teoría de los Ciclos Reales pero no deja de ser un reto considerable sobre todo para la cuestión que estamos considerando nosotros aquí, la de cual es el origen, la causa de las fluctuaciones. Si los shocks tecnológicos no producen un comovimiento entre el número de horas trabajadas y la productividad, que es lo que se deduce del esqueleto fundamental del modelo de RBC, su capacidad explicativa de los ciclos se reduce.

Una opción diferente es la de considerar que los cambios en la Productividad Total de los Factores son la causa de las fluctuaciones, pero que no actúan solos sino en conjunción con otras modificaciones que refuerzan sus efectos. De esta manera, las fluctuaciones cíclicas se explicarían por modificaciones significativas, pero no muy grandes, de la Productividad Total de los Factores acompañadas en cambios en la utilización de los factores de producción. La forma estándar de medir la PTF implica que la aportación de los factores al crecimiento de la producción es función directa de la cantidad de los mismos y su ponderación en la función de producción. Implícitamente se 
asume que sólo la cantidad tiene importancia, y no su grado de utilización ni su eficiencia, en gran parte porque resulta muy difícil medir en el agregado estos conceptos.

Burnside, Eichenbaum y Rebelo han explorado estos aspectos de los orígenes del ciclo económico en sendos artículos en 1993 y 1996. En el primero analizan la influencia de la acumulación de trabajo en el comportamiento de los ciclos económicos. En su modelo la función de producción, Cobb-Douglas, depende de la cantidad de capital y trabajo y del trabajo efectivo, entendido como esfuerzo por parte de los trabajadores. Correspondientemente la utilidad depende negativamente de ese esfuerzo. En su especificación la producción puede incrementarse tanto al aumentar la cantidad de trabajadores como su esfuerzo. Las empresas deciden la cantidad de trabajadores que quieren contratar de acuerdo con el estado de la tecnología y la demanda. Cuando se produce un shock las empresas se ajustan solicitando un cambio en el esfuerzo de los trabajadores que es compensado en términos de la desutilidad que implica ( Burnside, Eichenbaum y Rebelo 1993:249). De esta forma, el cambio en el esfuerzo laboral es procíclico, y contribuye a explicar parte del comportamiento de la producción. Como consecuencia, un shock tecnológico más pequeño, medido por la PTF puede explicar un cambio mayor en la producción, puesto que viene acompañado de una modificación en el nivel del esfuerzo laboral ${ }^{13}$.

De la misma forma los autores tratan la utilización del capital en su artículo de 1996. En este parten del análisis del residuo de Solow como medida de la PTF para señalar que se da por supuesto que los servicios productivos que rinde el capital se suponen proporcionales a la cantidad del mismo y proponen una alternativa diferente. De acuerdo con una tecnología del tipo de Leontief, indican que ${ }^{14}$ la producción depende de la combinación de materiales y valor añadido. Este último se obtiene combinando horas de trabajo con servicios de capital. En este caso los autores no introducen la posibilidad de que las horas de trabajo se maticen por el esfuerzo, sino que se concentran en los servicios del capital, en lugar de la cantidad del mismo. Para medir los servicios del capital asumen, basándose en estudios previos, que el consumo total de electricidad es proporcional a los servicios del capital. De esta manera incorporan la utilización del capital en la producción, en lugar de dar por supuesto que es igual a la cantidad del mismo. Al hacerlo así, como en el caso anterior, pueden explicar una parte más grande de la volatilidad en la producción, la variación cíclica, en términos de esa variable, y los

${ }^{13}$ Los autores señalan que tan sólo la mitad de la fluctuación se debe al shock tecnológico y la otra mitad al cambio en el esfuerzo ( Burnside, Eichenbaum y Rebelo 1993: 247)

14 Burnside, Eichenbaum y Rebelo (1996: 863) 
efectos de la PTF son más pequeños. En sus estimaciones la influencia de los shocks de productividad se reduce hasta en un $70 \%$ con respecto a los análisis tradicionales, lo que implica que los cambios en la PTF pueden ser del $30 \%$ de lo estimado previamente para dar lugar a la misma amplitud cíclica.

Una aproximación diferente a la cuestión es la de Greenwood, Hercowitz y Krusell (1997). Partiendo del hecho de que existe una relación negativa entre los precios de los nuevos bienes de capital de equipo y la cuantía de la inversión en los mismos, los autores sugieren que la inversión no general sino específica en nuevos bienes de equipo puede explicar buena parte de las fluctuaciones de la economía norteamericana entre 1954 y 1990. El modelo que especifican funciona bien para los años citados y ayuda a entender el ciclo. Su base consiste en que la reducción de los precios de los nuevos bienes de equipo permite a las empresas reducir el coste de uso de los mismos así como de los antiguos, porque se hace más barato reemplazarlos y por tanto explica incrementos sustanciales de la productividad que incentivas a las empresas a incrementar la inversión en ese tipo de bienes de equipo. El ejemplo al que se refieren concretamente son las computadoras. El problema fundamental de este modelo para nuestro propósito consiste en que puede servir para explicar la correlación negativa entre precios del nuevo equipo y cantidad invertida así como las desviaciones por encima de la tendencia de la producción. Pero no puede dar cuenta de las desviaciones significativas por debajo de la tendencia, sino tan sólo y parcialmente de la reducción de la cuantía de la desviación positiva ${ }^{15}$. Por tanto no haremos mención a esta posibilidad como causa de la crisis en adelante.

Con los matices mencionados, la forma de analizar si los shocks tecnológicos han tenido un impacto significativo como causa de la crisis en España es acudir a datos de la Productividad Total de los Factores. En general los datos muestran que la PTF ha crecido en España a tasas muy reducidas y todos los autores ponen de manifiesto que ese es uno de los principales problemas de la economía española. Los datos que utilizamos aquí los hemos obtenido de AMECO, base de datos económicos y financieros de la UE para sus países miembros y se presentan en forma de número índice con base 100 para el año 2000. Los datos de 2003 a 2007 son los siguientes.

15 Esta es una variante del problema general que tiene la RBC con lo que se denomina el «technological regress». Es difícil acomodar en un modelo de ciclos un cambio tecnológico negativo, sea general o parcial. 
Tabla 1. PTF en España (2003-2007)

\begin{tabular}{|c|c|c|c|c|c|}
\hline AÑ & 2003 & 2004 & 2005 & 2006 & 2007 \\
\hline PTF & 99,5 & 99,3 & 99,3 & 99,3 & 99,1 \\
\hline
\end{tabular}

De lo expuesto más arriba queda claro que los cambios en la Productividad Total de los Factores miden el efecto de los shocks tecnológicos. También ha quedado claro que el dato bruto de la PTF ha de ser matizado al menos por la utilización del capital y el esfuerzo laboral, aspectos que la cantidad de trabajadores y capital que se tienen en cuenta para calcular la PTF no miden bien. Por tanto un shock tecnológico habría de venir reflejado por un cambio en la PTF, pero ser menor que la misma, puesto que la PTF no refleja los matices a los que he hecho referencia antes. En consecuencia, si un shock tecnológico es la causa de la crisis habríamos de observar una variación de la PTF significativa, auque no del mismo tamaño que la fluctuación en la producción. Lo que se observa en los datos es algo bastante diferente. Por un lado, y a partir del valor 100 del año 2000 la PTF ha ido disminuyendo de forma continua desde ese año a 2007. Luego la PTF ha disminuido mientras los ritmos de crecimiento del PIB español se situaban por encima del 3\%. Si damos por supuesto que ese ritmo de crecimiento es la tendencia del PIB español en los últimos 10 años, cosa que es correcta como ponen de manifiesto los datos, lo que tenemos es una situación estable con una ligera disminución de la PTF. Pero precisamente por eso habríamos de esperar una fuerte y drástica reducción de la PTF en 2007 como expresión del shock tecnológico negativo que estaría en el origen de la crisis. Pero lo que muestra la PTF es la continuación de la reducción de la misma, casi al mismo ritmo que en años precedentes. La misma reducción que se da entre 2006 y 2007 se da entre 2003 y 2004, cuando la economía mantenía su ritmo de crecimiento. Por tanto la PTF no muestra ningún signo de shock tecnológico negativo, aunque sea pequeño. Los datos nos llevan a rechazar la hipótesis de que la crisis en España se haya debido a un shock tecnológico en sentido amplio.

\subsection{Los precios del petróleo}

El petróleo es una materia prima esencial cuyos precios históricamente han variado mucho. Por ambas razones, los incrementos en los precios del petróleo se consideran importantes para explicar factores macroeconómicos como la inflación, la estanflación o las crisis económicas, como la que nos ocupa aquí. La evidencia para los Estados 
Unidos muestra ${ }^{16}$ que hay una fuerte relación entre los períodos de crecimiento significativo de los precios del petróleo y las recesiones, aunque no siempre el cambio en los precios precede a la recesión.

El argumento teórico subyacente es que el incremento de los precios del petróleo reduce, vía elasticidad de la demanda, su consumo y dado que el petróleo es un insumo fundamental en la producción se reduce también la producción. Por ejemplo Rotemberg y Woodford (1996) muestran que para la economía de USA un incremento del $10 \%$ en los precios del petróleo reduce la producción en algo menos del 0,4\%, cifras pequeñas pero significativas. Por otro lado, se aduce que el incremento de los precios del petróleo conlleva unas transferencias de renta que reduce la riqueza de los habitantes del país. Dependiendo de hasta qué punto la economía de un país depende del crudo importado, esa pérdida de riqueza puede ser más o menos considerable ${ }^{17}$.

Para analizar el caso de España hemos recurrido a la serie de precios del barril de Brent en Euros $^{18}$.

Tabla 2. Precio del barril de Brent en Euros

\begin{tabular}{|c|c|c|c|c|c|c|c|c|c|c|}
\hline Mes & Mar & Jun & Sep & Dic & Mar & Jun & Sept & Dic & Mar & Jun \\
& 06 & 06 & 06 & 06 & 07 & 07 & 07 & 07 & 08 & 08 \\
\hline Precio & 51,6 & 54,1 & 48,7 & 47,3 & 46,8 & 52,9 & 55,4 & 62,4 & 66,8 & 85 \\
\hline
\end{tabular}

Los datos muestran que el precio del petróleo en este período ha crecido, pero lo ha hecho sobre todo a partir de Diciembre de 2007 y en adelante hasta llegar a un máximo en Junio de 2008. Después el precio ha flexionado a la baja y por ejemplo en Septiembre estaba ya en 68 Euros por barril y después ha seguido bajando situándose por debajo de los 50. Si suponemos que la crisis económica se gestó a lo largo de 2007 para hacerse evidente en 2008 tal y cómo indican los datos, se comprueba que el precio del barril entre Marzo y Diciembre de 2007 se incrementó más de un 30\% por lo que podría ser un factor fundamental en la misma. Pero cuando ampliamos la muestra de

16 Barsky y Kilian (2004)

17 Olson (1988) calculó valores en torno al 1\%-2\% del PIB para los países industrializados como consecuencia de la subida de precios de 1982-1985

18 Tomada de la Energy Information Administration of U.S. Government. Se aplican tasas de cambio mensuales entre el Dólar y el Euro y un deflactor basado en el IPC harmonizado tal y cómo lo publica Eurostat. 
los datos podemos observar que en Diciembre de 2004 el precio era de 29,5 Euros y en Septiembre de 2005 de 51 Euros, un incremento de más del 60\% en un lapso de tiempo similar. Ese incremento no conllevó una desaceleración de la actividad económica, pasando el crecimiento del PIB en tasa trimestral del 3,5\% al 3,6 \% . Si aceptamos que un fuerte incremento del precio del petróleo causa la recesión el mismo incremento, mayor, debería haberla causado en 2005, cosa que no ocurrió. Además, y como es obvio, el desplome de los precios actuales, que revierte los datos iniciales, debería conducir a una recuperación, y el PIB no muestra ese comportamiento. Por tanto, y aunque los precios del petróleo pueden tener un efecto en la actual recesión, ese efecto debe matizarse mucho, porque en otras circunstancia, es decir, con valores diferentes del resto de las variables, no ha generado recesión. Por tanto el shock del precio del petróleo ha de ser descartado como causante fundamental de la recesión.

\subsection{Shocks monetarios}

La Teoría de los Ciclos Reales ha ido evolucionando y se ha convertido en algo más general, un marco de análisis de la dinámica económica que puede utilizarse en diversos campos. Al hacerlo así, ha desbordado su objeto original de estudio, los ciclos económicos y se ha extendido por otros campos. Por la misma razón, y puesto que la metodología se ha utilizado profusamente, el carácter exclusivamente real de los shocks que fue el origen de la teoría ha dado paso a la posibilidad de considerar también algunos shocks de origen monetario. En este punto la influencia de los mecanismos monetarios en las fluctuaciones cíclicas se ha introducido con un carácter claramente keynesiano, que tiene en cuenta las posibilidades de rigideces, sobre todo en precios y salarios. La idea es que pueden darse shocks monetarios que de forma directa o indirecta tengan influencia en la evolución del ciclo económico, pero que no se trate de un mecanismo causal endógeno, que por consiguiente habría de generar ciclos de forma continua, sino de auténticos episodios puntuales que se superponen y modifican el funcionamiento de la economía, que la sacan de la tendencia que exhibe.

En un artículo de 2003, Galí, López-Salido y Vallés analizan las respuestas sistemáticas de la Reserva Federal de Estados Unidos a los shocks tecnológicos. Partiendo de los shocks tecnológicos presentes en la economía de Estados Unidos de 1954 a 1998 lo que estudian es de qué manera la política monetaria modificó, amplificó o redujo sus efectos sobre la economía, y de qué manera evolucionó a lo largo del tiempo esa política. Muestran a partir de los datos y con su modelo que durante el período en el que Paul Volcker y Allan Greenspan estuvieron al cargo de la Reserva Federal, la política 
se orientó a estabilizar al máximo posible los precios de acuerdo con una regla óptima de comportamiento de forma que los efectos sobre la producción fuesen los que se derivan del shock tecnológico, pero no más allá. El punto de partida son también los shocks tecnológicos, de cuya presencia no hemos encontrado rastro alguno para el caso de España en la actual crisis, por lo que este modelo no nos puede ayudar. Además y como es bien sabido la Reserva Federal tiene como objetivos tanto los precios como la producción, mientras que en el caso de la zona Euro el único objetivo explícito del Banco Central Europeo es el control de la inflación. Por tanto el análisis no puede aplicarse a nuestro caso.

Otra posibilidad de shock monetario consiste en el incremento de los tipos de interés, en este caso del tipo que aplica el Banco Central Europeo a toda el área de la zona Euro. A este respecto es necesario introducir múltiples cautelas. Está establecida una relación negativa entre el tipo de interés y la producción en términos teóricos pero los datos empíricos requieren matizarla. El Banco Central Europeo ${ }^{19}$ ha realizado exhaustivos estudios estadísticos sobre el efecto del incremento de los tipos de interés de un $1 \%$, mantenidos durante al menos dos años, sobre la producción agregada ${ }^{20}$. De los estudios econométricos se desprende que, bajo distintas especificaciones, que corresponden a distintas estructuras económicas, el efecto es de una reducción de la producción de en torno a un $0,3 \%$ el primer año, un $0,3 \%-0,7 \%$ el segundo y valores similares para el tercer año.

Los datos muestran que el tipo de interés del Banco Central Europeo subió del 3,5\% al 4,\% a principios de 2007 para mantenerse en ese nivel hasta Julio de este año en el que repuntó al 3,25\%. En Diciembre de 2008 se encuentra en un nivel del 2,5\%. Si aplicamos a estos datos la evidencia econométrica mencionada en el párrafo anterior deberíamos esperar una reducción de la tasa de crecimiento en España de 0,3 puntos porcentuales a comienzos de 2008, siendo el efecto superior a principios de 2009 . Como sabemos, la tasa de crecimiento española ha caído mucho más que esa reducción del 0,3 a comienzos de 2008 , hemos pasado de una tasa interanual del 4,0\% a otra del 2,6\%, por lo que si la política monetaria restrictiva del Banco Central Europeo ha tenido algún efecto ha sido muy pequeño, siendo el efecto fundamental como consecuencia de otras variables. Además el estudio mencionado especifica que esos son los valores

19 Hay trabajos similares para las políticas monetarias de la Reserva Federal, pero las diferencias entre el Banco Central Europeo y la Reserva Federal en cuanto a cometido, obligaciones y estructura son lo suficientemente grandes como para no aplicarlos directamente al caso de España.

${ }^{20}$ ECB's Monthly Bulletin, Octubre 2002. «Recent findings on monetary policy transmission». 
simulados de un incremento del tipo de interés que se mantiene durante dos años sin modificación alguna y al cumplirse esos dos años de forma aproximada ya hemos visto dos disminuciones sucesivas que han llevado el tipo de interés al 2,5\%. Por tanto podemos afirmar que el incremento del tipo de interés del Banco Central Europeo ha podido tener alguna influencia en la reducción de la producción española, pero muy pequeña en relación a la drástica reducción en la que nos encontramos. Si al cabo de dos años de incrementarse el tipo de interés la producción de la economía refleja una reducción del $0,7 \%$ del PIB en relación al escenario en el que ese incremento no se da, el crecimiento en tasa interanual del PIB en España en el primer trimestre de 2009 debería estar en torno al 3\%. Ese sería un resultado que en estos momentos no podemos ni soñar, porque el consenso de todos los expertos apunta a que nos encontraremos en recesión y en cifras muy bajas.

Otra opción distinta es la que proponen Bernanke, Gertler y Gilchrist ${ }^{21}$. Los autores señalan que las fricciones en el mercado de crédito amplifican los efectos de otros shocks en las fluctuaciones económicas. Su planteamiento de base es que en el proceso de intermediación entre el prestamista y el prestatario hay costes de agencia debido a la información asimétrica. Esos costes de agencia son menores cuanto mayor es la riqueza neta del deudor, porque en este caso compromete más parte de sus fondos propios en la inversión y por tanto sus incentivos se alinean mejor con los de quienes le prestan el dinero. Como consecuencia, cuanto mayor son los costes de agencia, menor la riqueza neta de los deudores, más restricciones al crédito hay y viceversa. Bajo el supuesto, plausible, de que la riqueza neta de los agentes es procíclica, lo que se deduce es que cuando la economía va bien las fricciones en el mercado de crédito son menores y se da un efecto de acelerador financiero que amplifica el efecto positivo de los shocks. Inversamente cuando la economía va mal la riqueza neta de los prestatarios se reduce, se incrementan las restricciones crediticias y el shock negativo resulta también amplificado. Por tanto en este caso el resultado es que un shock relativamente pequeño puede tener un efecto mayor debido al juego del acelerador financiero.

El análisis cuantitativo de los autores muestra que los efectos de una reducción no anticipada de 25 puntos básicos en el tipo de interés de los fondos federales, tiene, considerando un cierto retardo en la inversión, el efecto de incrementar la producción hasta un 0,8 por ciento al cabo de 3 trimestres. No desarrollamos más el modelo porque en el caso español, si bien el incremento del tipo de interés del Banco Central fue de 50 puntos básicos, como hemos puesto de manifiesto antes, de ninguna manera puede

${ }^{21}$ Bernanke, Gertler y Gilchrist (1999) 
suponerse que fuese no anticipado. El Banco Central Europeo había emitido suficientes señales para que el mercado fuese consciente de que el tipo de interés iba a subir. Por tanto los efectos son los de una subida anticipada, no es propiamente un shock. Eso por un lado. Por otro, la calibración que realizan los autores es para una disminución del tipo de interés, con el consiguiente incremento en la producción, mientras que en nuestro caso se trata de un incremento del precio del dinero y de una recesión posterior. Además el caso del Banco Central Europeo, como hemos puesto de manifiesto antes, no es el mismo que el de la Reserva Federal. Por si fuera poco, en el caso del trabajo de Bernanke et alii, lo que ellos consideran es un efecto de refuerzo de un shock ya existente. Lo que hemos mostrado hasta este punto es que las causas del shock negativo que se apuntan en la literatura no sirven para la crisis actual en España. No puede descartarse, puesto que no hemos demostrado que no haya ningún shock, que se haya producido un shock y que el mecanismo del acelerador financiero haya actuado, pero no tenemos apenas base para afirmar que ha tenido algo que ver.

\section{AlgunAs REFLEXIONES FINALES}

En este trabajo hemos abordado el análisis de las causas de la crisis en España, entendida como una fluctuación cíclica, desde la perspectiva de la Teoría de los Ciclos Reales, que es el estándar actual en el estudio de este tipo de fenómenos. Para ello hemos hecho referencia a una serie de trabajos de teóricos de RBC que abordan la cuestión de las causas de las fluctuaciones con carácter general ${ }^{22}$.

La Teoría de los Ciclos Reales se define como una «teoría de los ciclos de crecimiento» porque da implícitamente por supuesto que las economías crecen y que por tanto las fluctuaciones que se observan son desviaciones con respecto a ese crecimiento que la tendencia representa bien ${ }^{23}$. De forma implícita eso implica que las desviaciones negativas - recesiones - han de ser pasajeras y la economía vuelve pronto a su nivel

22 No es el único enfoque posible para probar la adecuación de la Teoría de los Ciclos Reales y su poder explicativo en estos momentos. Más adelante será necesario estudiar si los comovimientos entre las series agregadas que se deducen de los supuestos de la Teoría de los Ciclos Reales se dan o no en la presente crisis. El testeo de los mecanismos de transmisión tiene también un gran interés en estos momentos.

${ }^{23}$ No puede ser de otra manera puesto que parten del esquema de crecimiento económico de Solow. 
inicial puesto que los agentes reaccionan con rapidez para reajustar sus elecciones al nuevo entorno económico por lo que el equilibrio se reinstaura sin apenas dificultades. En estos momentos el carácter pasajero, y por tanto de fluctuación, de la situación económica está claramente en duda, aunque todavía no tenemos datos para afirmar nada diferente.

Cuando revisamos las explicaciones, las causas del ciclo según la RBC vemos que los shocks tecnológicos son la primera y más importante razón que perturba la economía. De forma plena, como planteaba Prescott (1986), o de forma más matizada por consideraciones acerca de la utilización de la capacidad productiva y de la acumulación de trabajo que hemos analizado en los trabajos de Burnside et alii (1993/1996) y otros, el shock tecnológico es un actor fundamental en el desarrollo discursivo de la teoría. Incluso, como hemos visto, desde otras perspectivas, los shocks tecnológicos pueden ser amplificados o matizados por la política monetaria, o acompañados por shocks monetarios en un sentido u otro. Por tanto el argumento fundamental se basa en la capacidad explicativa de los shocks tecnológicos que se miden por variaciones en la Productividad Total de los Factores. Para el caso español hemos mostrado que la PTF apenas varía, el cambio es difícil de distinguir estadísticamente de cero. Aunque se acepten los planteamientos de Burnside et alii, tiene que haber un cambio significativo en la Productividad Total de los Factores para que un shock tecnológico sea un buen candidato a causa de la actual recesión en España. Y ese cambio no se da. Por esa razón no hemos querido entrar en analizar la utilización de la capacidad productiva por medio del consumo de electricidad ${ }^{24}$, o en análisis de la mayor o menor utilización del esfuerzo laboral, porque las variaciones en la Productividad Total de los Factores son tan insignificantes que por muchos matices que se introduzcan no pueden explicar la recesión. Por tanto podemos descartar el shock tecnológico como causa de la crisis en España.

En segundo lugar hemos visto que el incremento del precio del petróleo tampoco nos puede explicar el fenómeno que estamos viviendo. De acuerdo con Barsky (2004) los efectos en la economía de los Estados Unidos son significativos, aunque no muy grandes. El análisis de los precios del petróleo en Euros muestra un incremento del $30 \%$ justo en las fechas previas al comienzo de la crisis, durante el año 2007. Pero si consideramos que un incremento de esa magnitud es el que causa la crisis nos encon-

${ }^{24}$ Las estadísticas del Banco de España muestran que el Consumo interior neto era de 24000 millones de KWh en Julio de 2007 y de 22000 en Julio de 2008. En Agosto de 2008 se consumió más electricidad (23043 millones de Kwh) que en Agosto de 2007 ( 22515 millones de Kwh) 
tramos que entre 2004 y 2005 el incremento fue casi del doble en términos porcentuales y la economía creció de forma homogénea. No podemos afirmar que el incremento del precio del barril de petróleo está en el origen de la crisis por sí sólo porque en este caso hubiéramos tenido ya una crisis a finales de 2005 y comienzos de 2006, cosa que no ha ocurrido. El precio del barril de petróleo muy posiblemente ha coadyuvado a desatar la crisis, pero por sí sólo no puede generarla.

En tercer lugar hemos pasado a examinar posibles shocks monetarios tal y cómo se interpretan desde la Teoría de los Ciclos Reales de los Negocios. Esta acotación es importante. La RBC considera que los shocks son exógenos y en gran medida inesperados, o al menos incontrolables, por lo que no pueden ser producto de la actividad «normal» de la economía. Además, los comienzos de la Teoría del Ciclo Real de los Negocios están relacionados con la negativa a considerar los factores monetarios como causantes del ciclo ${ }^{25}$. Por tanto este segundo conjunto de posibles causas es una elaboración que se aleja algo del núcleo central de los supuestos de partida de la RBC, y tiene que ver con la utilización de la metodología RBC como estándar para analizar la dinámica económica. Se trata fundamentalmente de modelos que tienen en cuenta los factores monetarios como amplificadores o difusores de otros shocks, y que no explican hablando propiamente el origen del ciclo como tal. Galí et alii (2003) se refieren al caso de Estados Unidos y parten de la existencia previa de un shock tecnológico cuyos efectos pueden ser modulados por la política de la Reserva Federal. En nuestro caso la inexistencia de un shock tecnológico, que está bien documentada, nos impide elaborar más el argumento de los autores. Por otro lado, el planteamiento del acelerador financiero de Bernanke et alii (1999) se aplica, en términos generales a cualquier tipo de shock aunque no esté definido. Eso abre la posibilidad de que el acelerador haya amplificado un shock cuyo origen no hemos especificado, pero la indicación de que la política monetaria pasa por los efectos de un cambio inesperado en los tipos de interés, echa por tierra sus posibilidades explicativas. Finalmente hemos planteado la posibilidad de que, sin modelo teórico alguno, el incremento del tipo de interés del Banco Central Europeo del 3,5\% al 4\% hubiera tenido algún tipo de incidencia. A falta de modelo explicativo hemos recurrido a los estudios econométricos del propio Banco Central que ponen de manifiesto que el efecto esperado es mucho más pequeño que el que se ha dado. De acuerdo con este análisis, y llevándolo al extremo, parte de la crisis podría achacarse a ese cambio en el tipo de interés, pero muy pequeña.

25 Kydland y Prescott (1994) 
En conjunto nos encontramos que las causas de las fluctuaciones económicas que se aducen en la literatura de la Teoría del Ciclo Real de los Negocios no nos explican la actual fluctuación negativa. Esta es nuestra principal conclusión. Es necesario buscar en otra parte las causas de la crisis en España porque las que los autores que se incluyen dentro de la RBC no pueden explicarla con los datos de los que disponemos. Ninguno de los shocks que se indican como causas de la fluctuación actúa en el caso español, y los factores monetarios, sobre todo las subidas de tipo de interés ni sirven como causas ni acompañan a otros shocks que se puedan identificar. La Teoría del Ciclo Real de los Negocios no nos sirve para explicar porqué se ha producido esta crisis.

La segunda conclusión se refiere a la importancia del análisis teórico de las causas de las fluctuaciones. El planteamiento de la RBC hace hincapié en que las causas son shocks exógenos y que por ello lo más importante es analizar los mecanismos de transmisión. Por ejemplo al estudiar la Gran Depresión, Prescott (1999) afirma que lo más importante es porqué la Gran Depresión se extendió durante tanto tiempo y tuvo la profundidad que tuvo mientras que las causas le parecen menos importantes. Al considerar que las causas son siempre shocks externos, para los teóricos de la RBC es más significativo el análisis de los comovimientos de las series en general y que los modelos explicativos den razón del sentido y la fuerza de las correlaciones que se observan en la realidad. Implícitamente se da por supuesto que las causas de las fluctuaciones no son importantes porque no pueden atribuirse a ninguna regularidad y por tanto el análisis económico puede decir poco acerca de ellas. Además el pre-supuesto de que los ciclos lo son de equilibrio, conduce a considerar que las fluctuaciones son un desequilibrio que se reequilibrará sólo en un lapso relativamente pequeño de tiempo. Precisamente el gran problema que presenta la Gran Depresión a los teóricos de RBC es su duración, porque si los ciclos son de equilibrio, lo cual repito es un pre-supuesto, la economía debería volver al equilibrio con rapidez.

Minusvalorar la importancia del análisis de las causas de las fluctuaciones presenta dos problemas principales. El primero es que desde el punto de vista teórico la economía ha de explicar, y no tan sólo predecir. Es una tarea del teórico explicar lo que ocurre en base a los conocimientos y datos de los que se dispone, y no se puede abdicar de esa tarea. Es evidente que si las fluctuaciones no presentan un comportamiento periódico esa ausencia de regularidad hace el trabajo más difícil pero no por eso puede ser eludido. Explicar siempre es importante. El segundo problema es que saber porqué se dan las fluctuaciones cíclicas es necesario para poder actuar para corregirlas. De nuevo la RBC con su planteamiento de ciclos de equilibrio da por supuesto que no es preciso corregir nada y que no se puede corregir nada o casi nada en las fluctuaciones. Por ejemplo Prescott y otros teóricos achacan la duración de la Gran Depresión en gran medida a la mala intervención del sector público en Estados Unidos y afirman que sin 
esas actuaciones de política económica la crisis hubiese durado menos y hubiese sido de menor calado. Pero eso, de nuevo es un pre-supuesto. Si damos por sentado que la economía vuelve al equilibrio por sí sola no es necesario corregir nada, pero es negativo darlo por sentado. Puede ocurrir que el reequilibrio sea automático, pero puede ocurrir que no lo sea, por lo que necesitamos saber qué causa la o las crisis económicas, las fluctuaciones de una cierta entidad. Quizá la causa, el shock exógeno en la terminología de la RBC sea diferente en una fluctuaciones u otras, pero es razonable pensar que hay una cantidad limitada de posibles causas de las fluctuaciones, y que el análisis de las causas de cada una de esas fluctuaciones nos permitirá llegar a un conocimiento de los mecanismos de casi todas ellas, si no de todas. Eso no implica que sea necesaria la intervención para corregirlas, porque algunas de ellas conllevarán su propio mecanismo de reequilibrio, pero otras no, y no se puede dar por supuesto a priori que eso es así con carácter general.

Por lo tanto de nuestro trabajo se deducen dos ideas fundamentales. La primera que la Teoría de los Ciclos Reales no explica la actual crisis económica en España. Es necesario e imprescindible investigar las posibles causas desde otras perspectivas teóricas, sean las que sean, porque la Teoría de los Ciclos Reales no sirve para ello. Como consecuencia, los teóricos de la RBC y todos los que trabajan dentro de su marco metodología han de plantearse la cuestión de la explicación de las fluctuaciones no como algo secundario, sino central en la investigación. En segundo lugar pone de manifiesto, con carácter más general, que la teoría económica ha de buscar explicaciones a los fenómenos, aunque en ocasiones sean difíciles. La creación de modelos que puedan simular bien la evolución de los datos empíricos disponibles es muy importante porque permite conocer bien las relaciones entre las variables, pero no es suficiente. Es imprescindible saber porqué se dan los fenómenos, sobre todo en el caso de fenómenos agregados de la dimensión de las fluctuaciones económicas. La Teoría de los Ciclos Reales queda coja si no aborda esa cuestión.

\section{REFERENCIAS BIBLIOGRÁFICAS}

Barsky, R. y Kilian, L. (2004), «Oil and the Macroeconomy since the 1970s», Journal of Economic Perspectives, otoño, núm. 18, 4, págs. 115-134.

Bernanke, B.; Gertler, M. y Gilchrist, S. (1999), «The financial accelerator in a quantitative business cycle framework», en J. Taylor y M. Woodford, Handbook of Macroeconomics, vol 1C, Amsterdam, North Holland.

Burns, A. y Mitchell, W. (1946), Measuring business cycles, Nueva York, NBER. 
Burnside, C.; Eichenbaum, M. y Rebelo, S. (1993), «Labor Hoarding and the Business Cycle», Journal of Political Economy, vol. 101, núm. 2, págs. 245-273.

- (1996), «Sectoral Solow residuals», European Economic Review, vol. 40, págs. 861-869.

GaLí, J. (1999), «Technology, Employment, and the Business Cycle: Do Technology Shocks Explain Aggregate Fluctuations?», The American Economic Review, núm. 89, 1 págs. 249-271.

Galí, J.; LóPeZ-SAlido, D. y VAllÉs, J. (2003), «Technology shocks and monetary policy: assessing the Fed’s performance», Journal of Monetary Economics, núm. 50, págs. 721-743.

Greenwood, J.; Hercowitz, Z. y Krusell, P. (2000), « The role of investment-specific technological change in the business cycle», European Economic Review, núm. 44, págs. 91-115.

Kydland, F. y Prescott, C. (1982), «Time to build and Aggregate Fluctuations», Econometrica, 50, 6, págs. 1345-1370.

Kydland, F. y Prescott, E. (1994), Business Cycles: Real Facts and a Monetary Myth, Federal Reserve Bank of Minneapolis.

Lucas, R. (1977), «Understanding business cycles», en Karl Brunner y Allan H. Meltzer (ed.), Stabilization of the domestic and international economy, Carnegie-Rochester Conference Series on Public Policy, Amsterdam Norh-Holland.

Mankiw, G. (1989), «Real Business Cycles: A New Keynesian Perspective», Journal of Economic Perspectives, vol. 3, núm. 3, págs. 79-90.

Olson, M. (1988), «The Productivity Slowdown, the Oil Shocks and the Real Cycle», Journal of Economic Perspectives, otoño, 2, págs. 43-69.

Prescott, E. (1999), «Some observations on the Great Depresion», Federal Reserve Bank of Minneapolis Quaterly Review, núm. 23, págs. 25-31.

- (1986), «Theory Ahead of Business Cycle Measurement», Carnegie-Rochester Conference Series on Public Policy, 25, págs. 11-44.

Rotemberg, J. y Woodford, M. (1996), «Imperfect Competition and the Effects of Energy Price Increases on Economic Activity», Journal of Money, Credit and Banking, noviembre, núm. 28, págs. $550-577$.

Solow, R. (1956), «A contribution to the theory of economic growth», Quarterly Journal of Economics, 70, págs. 65-94. 